\title{
Cybercrime: youth awareness survey in Delhi NCR, India
}

\begin{abstract}
Nowadays cybercrime is rapidly increasing day by day with the evolution of technology where we take a step towards the digital world. With every step, new challenges and responsibilities are also arising daily basis. The concentrated population for the attack is Youth and teenagers. They are the major population of the world who are enjoying the digitalization. Cybercrime is a major threat to the growing society. Every country in the world promoting digitalization to survive in this digital world and to secure data government and non-government organizations taking responsibility to secure our data from the attackers. To secure government and other organizations' understanding and analyzing the things to secure data. In India, the majority of the population belongs to rural areas and they are the easy target for the attackers to manipulate. Many people don't know about cybercrimes and security, so did a survey in Delhi NCR, India to analyze about the cybercrime.
\end{abstract}

Keywords: cybercrime, cyber-attack, awareness, security
Volume 8 Issue 5 - 2020

\author{
Yogesh Meena,' Mahipal Singh Sankhla, ${ }^{2}$ \\ Shriyash Mohril, ${ }^{3}$ Rajeev Kumar ${ }^{2}$ \\ 'Students of Bachelor of Technology Computer Science and \\ Engineering, Galgotias University, India \\ ${ }^{2}$ Department of Forensic Science, School of Basic and Applied \\ Sciences, Galgotias University, India \\ ${ }^{3}$ Process Associate, Roboticwares Pvt. Ltd. Noida, India
}

Correspondence: Mahipal Singh, Department of Forensic Science, Galgotias University Greater Noida, India, Email mahipaln6@gmail.com

Received: August 23, 2020 | Published: October 15, 2020

\section{Introduction}

To a great extent of we have very restricted information on crime occurring in "cyberspace", known as cybercrime, which occurs on the computer and the Internet, however, cybercrime has a severe potential for a notable impression on the lives of people and our society. ${ }^{1}$ When we talk about the data and its protection, data constraint, and information security chances into a big worry. ${ }^{2}$ There is no uncertainty that nowadays practically all facility providers have made life relaxed by use of technology be it Banking, Insurance, Ecommerce, etc. technology not only links the distance but also delivers a healthier service to the clients. With the fast development of Information technology in business, consumers are now helpless to many dangers. ${ }^{3}$ New and cultured cybercrime tools could function to allow a nationstate or terrorist group to continue anonymously while they direct cyber-attacks over the Internet. ${ }^{4}$ Many professional's facts out that past event of conventional terrorism have already been connected with cybercrime, and that computer exposures may make administration and citizen serious infrastructure systems appear attractive as targets for cyberattack. ${ }^{5}$ The impression of cybercrime is not just evaluated only in terms of costs suffered but also in terms of the break of information privacy which can affect many consumers. The expected losses for the industries by the year 2019 due to cybercrime are projected to be in the area of $\$ 2$ trillion. ${ }^{6}$ While the number of cyber security outbreaks in big companies has been declining, in medium and small-sized companies it is growing expressively, which could be the main concern for developing nations.?

\section{Cyber crime}

In general, cybercrime may be defined as "Any illegal act where computer or communication device or computer network is used to promise or enable the command of crime". ${ }^{8}$ Around 3.24 million outbreaks were directed by cybercriminals in 2006. Some online crimes have even exceeded their counterparts in the physical world. In the meantime, specialists evaluate that near $90 \%$ of the cybercrimes go unreported. ${ }^{9}$ Cybercrime varies across a variety of events.
At one end, are crimes that include essential breaches of an individual or communal privacy, such as attacks on the integrity of data held in digital collections and the use of unlawfully found digital information to extortion a firm or an individual. At the other end of the variety are those misconducts that contain tries to interrupt the authentic mechanisms of the Internet. Cybercrimes can be mostly divided into four main categories. ${ }^{10}$

Cybercrimes against individuals: Cyber-crimes dedicated against people consist of numerous crimes like the broadcast of childpornography, cyber porn, the nuisance of an individual by a computer such as through e-mail, forged escrow scams. The transferring, spreading, posting, and dissemination of offensive material with pornography and indecorous exposure establishes one of the most significant Cybercrimes identified nowadays. ${ }^{3}$

Cybercrime against property: Another organization of Cyber-crimes is that, Cybercrimes against all forms of assets. These crimes include computer vandalism (obliteration of others' property), Intellectual Property Crimes, Intimidating, Salami Attacks. This type of crime is generally dominant in financial institutes or for the determination of obligating financial crimes. A vital feature of this sort of wrongdoing is that the alteration is so insignificant that it would usually go unnoticed. ${ }^{11}$

Cybercrime against companies/organizations: This is one of the most usual forms of cyber-crime today. When a company's online occurrence or any of its goods are hacked, it converts a serious problem that can consequence in a large number of penalties for the company, as well as their personnel, associates, and clients. Examples include information breaches, cyber squeezing and warez spreading, etc. ${ }^{12}$

Cybercrime against government: The evolution of the Internet has exposed that the standard of cyberspace is being used by individuals and groups to lurk the governments as also to threaten the peoples of a state. This crime demonstrates itself into violence when an individual hack into an administration or military preserved website. ${ }^{13}$ 


\section{Cyber attack}

Cyber Attack is done by cybercriminals. They use computer systems or networks to manipulate, steal, or gain unauthorized access to the data. Cyber Crime is a threat to information security due to growth towards digitization our main concern is to secure digital assets by Cybercriminals. They use different security tools and techniques to manipulate data.

\section{Material and methodology}

\section{Tools of data collection}

An instrument is assistance with required and related data that could be collected methodically to the subject material. Google Form was used to record responses. In a data set, we collected 151 responses based on that we conclude the data. The Survey process was the means for information gathering, which was used by the scholars for the determination of assembling information from the respondents. The Survey technique is the best way for gathering the maximum data in an organized technique. ${ }^{14}$

\section{Data collection and procedure}

The research is constructed on primary information gathering from Google form and the data for the study was acquired from the respondents. The information was composed by a simple random sampling process. Belief and understandings of the respondents were composed through the Survey method. The scholar after building an understanding with the respondents defined the determination, importance, and meaning of the study.

\section{Result and discussion}

In Figure 1 we show the age collection of respondents. The age of the respondents, $68.2 \%$ of the respondents belong to the age group of $16-21$ years, $29.1 \%$ of the respondents belong to the age group of $22-28$ years, only $2.6 \%$ of the respondents belong to the age group of 29-35 years.

\section{Your age group.}

151 responses

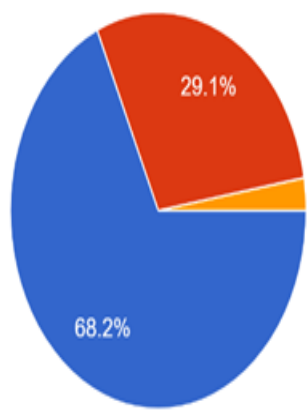

Figure I Age group of male \& females.

\section{Response based on gender}

In Figure 2 respondents were categorized according to gender. Respondents based on gender, $60.3 \%$ of the respondents were male and $39.3 \%$ of the respondents were female.

\section{Awareness about cyber crime}

In Figure 3 respondents were asked they know about Cybercrime/ Cyberterrorism, 98\% of the respondents were about Cybercrime/
Cyber terrorism, only 2\% of the respondents don't know about Cybercrime/Cyberterrorism.

\section{Gender}

151 responses

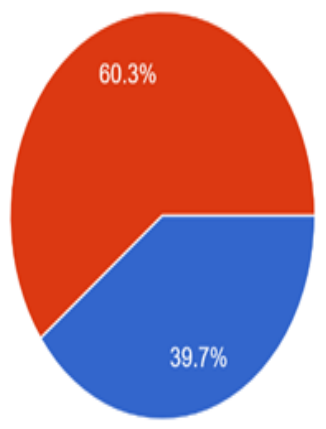

Female Male

Figure 2 Response based on gender.

\section{Do you heard about cyber crime/cyber terrorism?}

151 responses

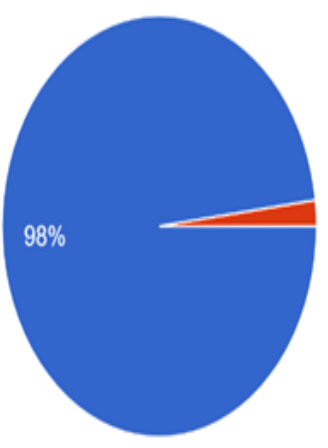

Figure 3 People awareness about cyber crime.

\section{Source of awareness about cyber crime}

In Figure 4 respondents were asked about from where they heard about cyber-crime in multiple-choice question form in which $78.8 \%$ of the respondents knew about this from internet, $41.00 \%$ of the respondents were heard from the friends, $22.5 \%$ of the respondents were heard from their family members, $49.00 \%$ of the respondents were read from newspaper, $47.7 \%$ of the respondents were heard from television, $18.5 \%$ of the respondents heard from any other sources.

Where do you heard about this?

151 responses

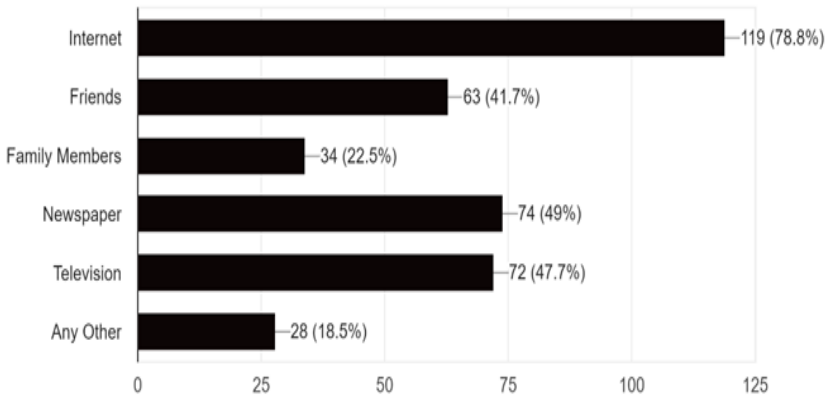

Figure 4 Source of awareness about cyber crime. 


\section{Awareness about cyber attack}

In the Figure 5 respondents were asked about them about cyberattack, $77.5 \%$ respondents were heard about cyber-attack, $16.6 \%$ of the respondent where they may be heard about cyber-attack, while $6 \%$ of the respondents about don't about the cyber-attack.

\section{Do you heard about any Cyber Attack? \\ 151 responses}

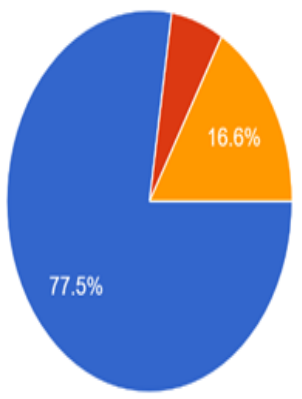

Figure 5 Awareness about cyber attack.

\section{Awareness of different cyber attacks}

In the Figure 6 respondents were asked about they know about cyber-attacks in multiple-choice question form in which $66.3 \%$ of the respondents know about Phishing, $15.9 \%$ of the respondents know about Eavesdropping attack, $20.5 \%$ of the respondents know about SQL Injection, $49.00 \%$ of the respondents know about Malware, $17.2 \%$ of the respondents know about DDoS, $57.0 \%$ of the respondents know about Spamming, $51.7 \%$ of the respondents know about Cyber Stalking, $44.4 \%$ of the respondents know about Software Privacy, $18.5 \%$ of the respondents know about Social Engineering, $49.7 \%$ of the respondents know about Computer Virus, $60.9 \%$ of the respondents know about Child Pornography, Only 3.3\% of the respondents don't know about any of the above cyber-attacks.

Select the common cyber attacks that you know

151 responses

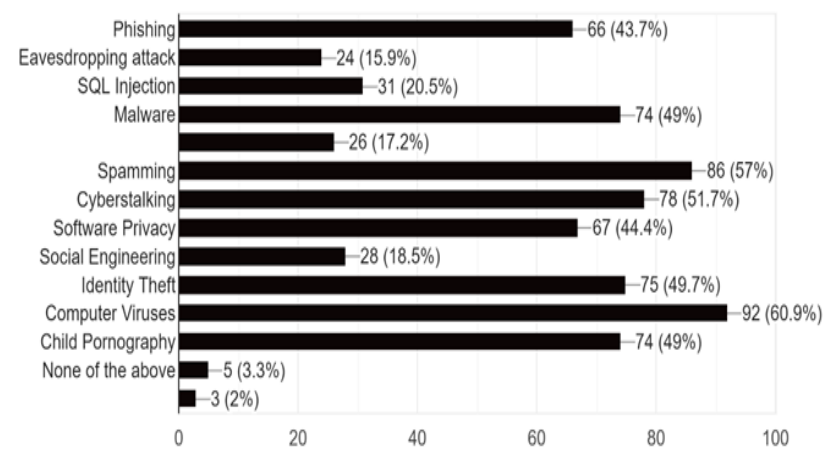

Figure 6 Awareness of different cyber attacks.

\section{Awareness about any agency for cyber security}

In the Figure 7 respondents were asked about they know the government or non-government or group of hackers related to cyber security, $43.7 \%$ of the respondents were know about agency related to Cyber security, while $56.3 \%$ of the respondents did not know about agency related to cyber security.
Do you know about any government organisation or non-government organisation or group of hackers related to cyber crime and security?

151 responses

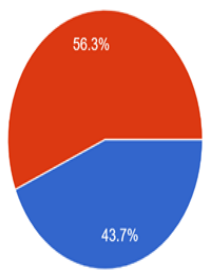

Yes

№

Figure 7 Awareness about any agency for cyber security.

\section{Awareness about major cyber attacks}

In the Figure 8 respondents were asked about from where they heard about major cyber-attacks in multiple-choice question form in which $33.1 \%$ of the respondents know about Wanna Cryransomware attack, $13.2 \%$ of the respondents know about Marriott Starwood data breach, $22.5 \%$ of the respondents know about Denial-of-Service attack, $34.4 \%$ of the respondents know about Yahoo data breach, $25.8 \%$ of the respondents know about South Korea Cyber Attack, $33.8 \%$ of the respondents don't know about any of the above-listed cyber-attacks.

Select the cyber attack you have heard about from the famous major cyber attacks that are listed below

151 responses

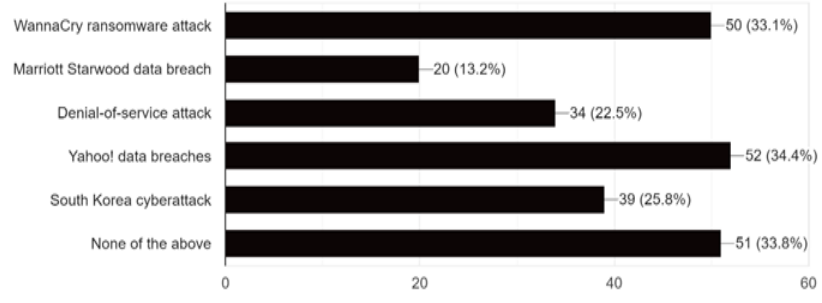

Figure 8 Awareness about major cyber attacks.

\section{Awareness about the prevention of cyber attack}

In the Figure 9 respondents were asked about from where they about in multiple-choice question form in which $45.0 \%$ of the respondents know about Firewall, 70.9\% of the respondents know about Antivirus/Malware Software, $13.2 \%$ of the respondents know about Penetration Testing, $23.2 \%$ of the respondents know about IDS, $9.3 \%$ of the respondents don't know about any of the listed security software.

Which security software/program do you prefer to prevent from cyber attack or viruses? 151 responses

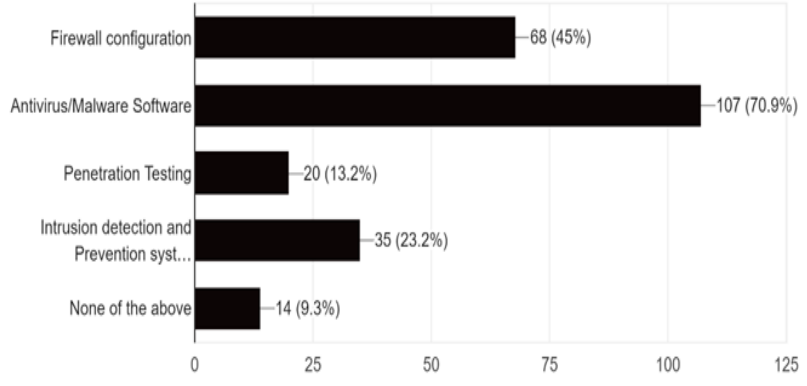

Figure 9 Awareness about the prevention of cyber attack. 


\section{Conclusion}

In the Google form, we have asked to mention suggestions from the respondents to spread awareness towards cybercrime and what they expect from the government and security experts to stop cybercrime. From the respondent's suggestions, we conclude their suggestions.

\section{What do you think about cybercrime and cyber terrorism and cyber security?}

There are so many different opinions about cybercrime crime terrorism and cyber security. We have recorded 91 responses out of 151 respondents.

Cybercrime is a criminal activity that is performed by the individual to steal information or to get unauthorized access to the system with the help of network and computer systems using hacking tools. Cyber terrorism is the illegal activity performed by a group of people over the internet to gain information from individuals or organizations to defame, destroy, or get important information. Cyber Security is the security to prevention from cybercrime and it is everyone's right to use fearless network and internet. Cybercrime is a loophole to Cyber Security. Respondents compare cyber war with physical war. The rapid growth of internet users adds risks to cyberspace and it can to too dangerous to normal internet users.

\section{Your suggestions how can we prevent cyber crime?}

Respondents have suggested many methods to prevent cybercrime. We have recorded responses out of 151 respondents.

Respondents were asked about how can we prevent cybercrime; respondents gave different suggestions to prevent cybercrime. They suggested that an awareness campaign should be started to spread awareness among people. Knowledge of information technology should be mandatory for all people. India is the 2nd largest number of internet users.

What do you expect from the government or other non-government organizations to reduce this type of cyber-attacks?

There are many different ideas and expectations of the respondents to reduce cyber-crime. We have recorded 77 responses out of 151 respondents. The government should make strict laws and regulations for crimes and start awareness programs in rural as well as urban areas because in rural areas people are most affected by spam calls and messages. Youths and aged people are easy targets for attackers. Organizations and experts make powerful tools and software to secure systems and information. A data breach is the main concern of the respondents due to the recent activities performed in the country. There should be an eye on suspicious activities on the internet and take action as soon as possible. Respondents take the major concern to suggestions on women safety, child pornography, and anti-national activities performed over the internet which damages our society, government and non-government organization should come to together to find the solution of such problems.

\section{Acknowledgments}

None.

\section{Conflicts of interest}

The authors declare there are no conflicts of interest.

\section{Funding}

None.

\section{References}

1. Jonathan Clough. Principles of Cybercrime. Cambridge, 2. 2010.

2. Dhruba KB, Kalita JK. DDoS Attacks: Evolution, Detection, Prevention, Reaction, and Tolerance. CRC Press/Taylor \& Francis Group; 2016.

3. Reddy KS. Cyber crimes in india and the mechanism to prevent them IJIRIS. 2014;9(3):29-32.

4. Tim Greene. Storm Worm Strikes Back at Security Pros. NetworkWorld. com. 2007.

5. Brian Krebs. Three Worked the Web to Help Terrorists. The Washington Post. 2007.

6. Juniper Research. Cybercrime will Cost Businesses Over $\$ 2$ Trillion by 2019 - Juniper Research. 2019.

7. Symantec. Attackers Target Both Large and Small Businesses. 2016.

8. https://cybercrime.gov.in/Webform/CrimeCatDes.aspx

9. Cyber Crime Overview. 2008

10. Wadhwa A, Arora N. A Review on Cyber Crime: Major Threats and Solutions. International Journal of Advanced Research in Computer Science. 2017;8(5).

11. Poonia AS. Cyber Crime: Challenges and its classification. International Journal of Emerging Trends \& Technology in Computer Science (IJETTCS). 2014;3(6):119-121.

12. https://securitytrails.com/blog/types-of-cyber-crime

13. https://www.legistify.com/qna/answer/1912-cyber-crime-againstgovernment/

14. Afrozulla KZ, Vaishnavi RT, Arjun. Cyber Crime Awareness among Msw Students, School Of Social Work, Mangaluru. J Forensic Sci \& Criminal Invest. 2018;9(2):555757. 